\title{
The effect of electric fields on nonaqueous dispersions
}

\author{
R. H. Ottewill ${ }^{1}$ ), A. R. Rennie ${ }^{2}$ ) and A. Schofield ${ }^{1}$ ) \\ $\left.{ }^{1}\right)$ School of Chemistry, University of Bristol, UK \\ ${ }^{2}$ ) Institut Laue Langevin, Grenoble, France
}

\begin{abstract}
Small angle neutron scattering has been used to investigate the structure of poly(methylmethacrylate) dispersions in dodecane in the presence of an electric field. In dodecane alone field effects were not observed. In the presence of calcium octanoate, however, the particles apparently become charged and increased ordering was observed. The effect of an electric field on the latter dispersions produced anisotropy of structure which suggested that the particles were forming loose "strings of beads" in the direction of the applied field.
\end{abstract}

Key words: Neutron scattering; polymer latices; nonaqueous dispersions; electric fields; microstructure

\section{Introduction}

The effects of electric fields on nonaqueous dispersions have been rather sparsely studied in comparison to studies carried out on aqueous systems. However, the availability of monodisperse spherical polymer particles at high volume fractions in media of low relative permittivity $[1,2]$ has provided excellent systems for fundamental studies $[3,4]$. Moreover, the development of small angle neutron scattering has provided a means of studying the structure of concentrated colloidal systems in applied fields and has already been used to examine systems of this type under shear fields $[5,9]$.

In this communication we provide a brief report on the use of small angel neutron scattering to investigate the effect of applied electric fields on the microstructure of dispersions of poly(methylmethacrylate) particles stabilised by poly (12-hydroxystearic acid) in a hydrocarbon medium. In the first instance the particles were examined in dodecane alone and then in dodecane with the addition of small amounts of calcium octanoate to provide some conductance to the dispersion medium.

\section{Experimental}

Materials

Dodecane was B.D.H. material.
NUOSYN CALCIUM 10\% was obtained from Durham Chemicals Limited, Birtley, Chester-le-Street, Co. Durham, UK. According to the manufacturers this is a solution of calcium iso-octanoates in a hydrocarbon medium. It is reported in this communication as calcium octanoate.

The polymer latex, SPSO90, was prepared by the dispersion polymerisation technique previously described [2]. The number average particle diameter determined by electron microscopy was $0.20 \mu \mathrm{m}$ and the weight average diameter determined by small angle neutron scattering was $0.22 \mu \mathrm{m}$ [5].

\section{Small angle neutron scattering}

The small angle scattering measurements were carried out using the neutron diffractometer D11, at the Institut Laue Langevin, Grenoble [10], with a sample-detector distance of $35.7 \mathrm{~m}$ and a neutron beam wavelength of $0.80 \mathrm{~nm}$. For elastic scattering measurements, the magnitude of the scattering vector, $Q$, is defined by,

$$
\mathrm{Q}=4 \pi \sin (\theta / 2) / \lambda
$$

with $\theta=$ the angle between the incident and the scattered beams.

The measurements were carried out in an optical-standard quartz cell with a pathlength of $1 \mathrm{~mm}$. Bright platinum rods were inserted into the cell at a separation distance of $6 \mathrm{~mm}$. An A.C. field if frequency $50 \mathrm{~Hz}$ was applied across the electrodes. The applied voltage was varied from zero to $1800 \mathrm{~V}$. The experimental arrangement is illustrated schematically in Fig. 1. The neutron beam, of diameter $4 \mathrm{~mm}$, passed between the electrodes in a direction perpendicular to the applied electric field.

The scattered neutrons were measured using a two-dimensional detector, a square matrix of $64 \times 64$ elements. 


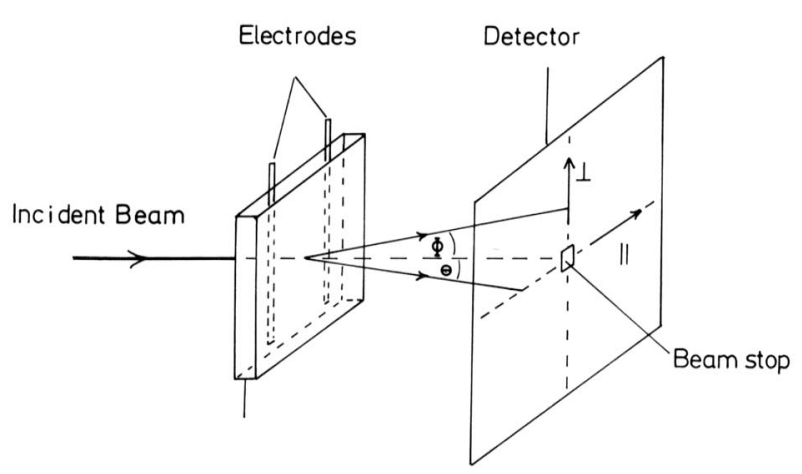

Fig. 1. Experimental arrangement used for examining the small angle neutron scattering from a polymer colloid dispersion during the application of an electric field

This allowed either two-dimensional contour plots to be obtained showing lines of equal intensity or three-dimensional plots showing intensity across the detector. For isotropic two-dimensional patterns the intensities can readily be radially averaged to give one-dimensional plots of intensit, $I(Q)$, against $Q$. Anisotropy if the sample can be clearly recognised in a contour plot. In this case in order to obtain plots of $\mathrm{I}(\mathrm{Q})$ against $\mathrm{Q}$ the averaging procedure can be restricted to small sectors, for example, parallel $\left(0 \pm 15^{\circ}\right)$ and perpendicular $\left(90 \pm 15^{\circ}\right)$ to the direction of the applied field [11].

\section{Results}

Intensity measurements without an electric field

The intensity of scattering at a particular value of $\mathrm{Q}$ is, for spherical particles, given by $[3,4]$,

$$
\mathrm{I}(\mathrm{Q})=\mathrm{A} \phi \mathrm{V}_{\mathrm{P}} \mathrm{P}(\mathrm{Q}) \mathrm{S}(\mathrm{Q})
$$

Where for spheres of the core-shell type A contains instrumental constants, and terms in coherent scattering length density described elsewhere, [3], $\mathrm{V}_{\mathrm{P}}=$ the particle volume, $\phi=$ volume fraction of the particles, $P(Q)=$ the particle shape factor and $S(Q)=$ the structure factor.

Figure 2 shows a curve for a $40 \%$ w/w dispersion of the latex SPSO90 in dodecane. This shows a broad peak consistent with short-range interactions of the hard sphere type examined in earlier work on polymethylmethacrylate latices [3-5]. The addition of calcium octanoate to the sample caused a substantial change in the form of the curve. As can be seen from Fig. 2, the broad peak of the original latex became very much narrower and sharper. Since the basic latex was the same in both cases, i.e. P(Q) was the same,

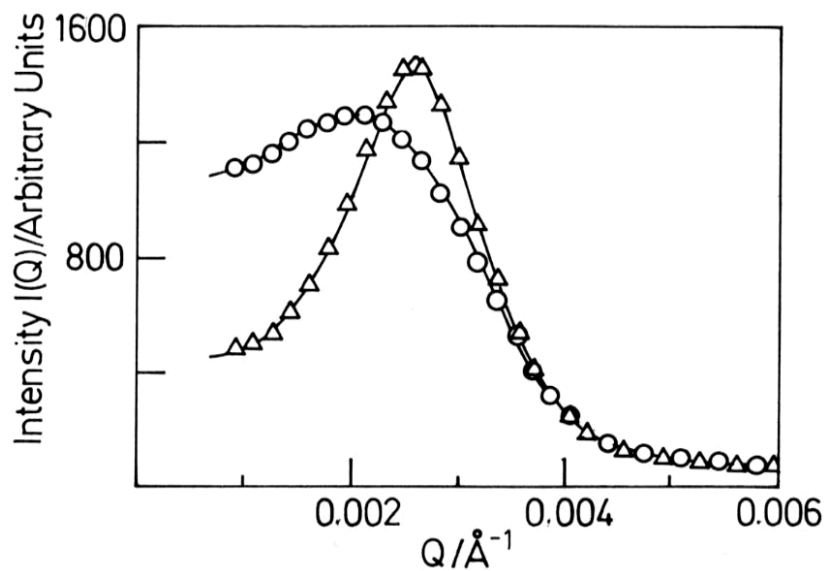

Fig. 2. Intensity, $I(Q)$, against scattering vector $Q$, for a $40 \%$ $\mathrm{w} / \mathrm{w}$ poly(methylmethacrylate) latex dispersion: -O-, in dodecane; $-\triangle-$, in dodecane containing $0.1 \%$ calcium octanoate

and the samples were essentially in the same medium, A also remained constant. Thus the changes in the scattering curve were attributed to changes in $S(Q)$. The changes in shape indicated an increase in interaction between the particles with the implication that this arose because the addition of calcium octanoate caused the particles to acquire a weak electrical charge. This led to the form of the interaction changing from that between sterically stabilised particles, a short-range repulsion, to that between electrically charged particles, a long-range repulsion. In the latter case the system becomes more ordered at the same volume fraction, and hence the peak in $I(Q)$ against $\mathrm{Q}$ becomes more clearly defined. Electrophoresis measurements on dilute dispersions confirmed that in the presence of calcium octanoate the particles had a positive mobility.

\section{The effect of an electric field}

\section{Calcium octanoate absent}

Figure 3 shows the 2-dimensional contour plots obtained using an $18 \% \mathrm{w} / \mathrm{w}$ dispersion of poly(methylmethacrylate) particles in dodecane alone. The pattern obtained from latex in the absence of the field is shown in Fig. 3a, and that with a field strength of $3800 \mathrm{~V}$ $\mathrm{cm}^{-1}$ applied is shown in fig. $3 \mathrm{~b}$. No discernable change in the pattern was observed on the application of the field. 


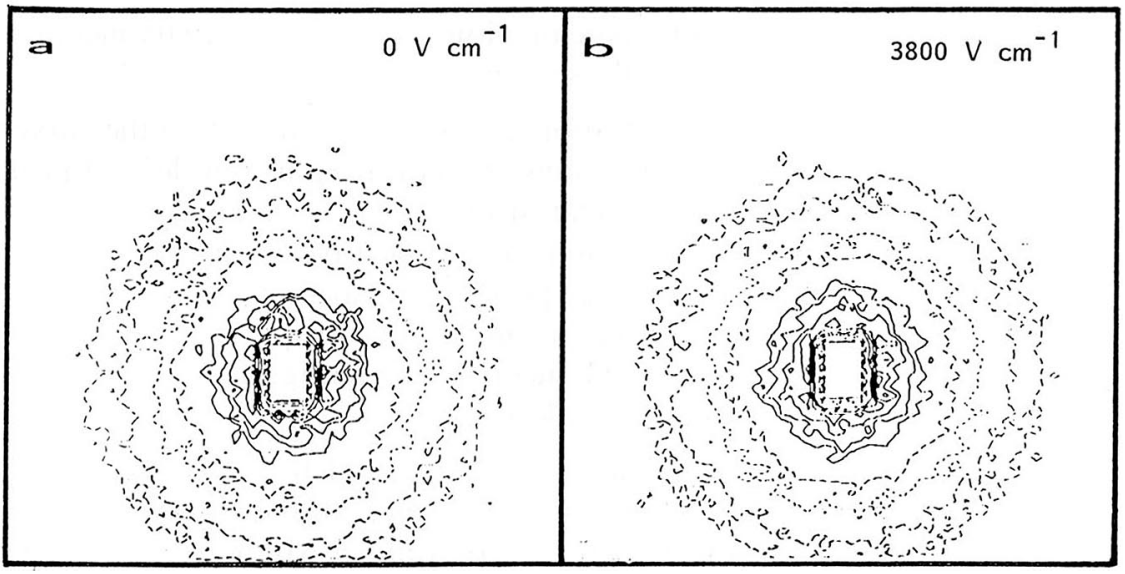

Fig. 3. Contour plots of constant intensity, $I(Q)$, lines against $Q$ for an $18 \% \mathrm{w} / \mathrm{w}$ poly (methylmethacrylate) dispersion in dodecane in the absence and presence of an electric field

\section{Calcium octanoate present}

Figure 4 shows the 2-dimensional pattern obtained using a $10 \% \mathrm{w} / \mathrm{w}$ dispersion of polymethymethacrylate) containing $0.025 \%$ calcium octanoate. Figure $4 \mathrm{a}$ shows the absence of an applied field. The contours are symmetrical showing that the sample is behaving as an isotropic dispersion with the peak in the structure factor showing up as a well-defined ring. Figures $4 \mathrm{~b}, 4 \mathrm{c}$ and $4 \mathrm{~d}$ illustrate the contour plots obtained at field strengths of 900,1800 and $3800 \mathrm{~V} \mathrm{~cm}^{-1}$. The anisotropy of the sample increases as the field strength increases and is most marked in Fig. 4d the system at the highest field strength.

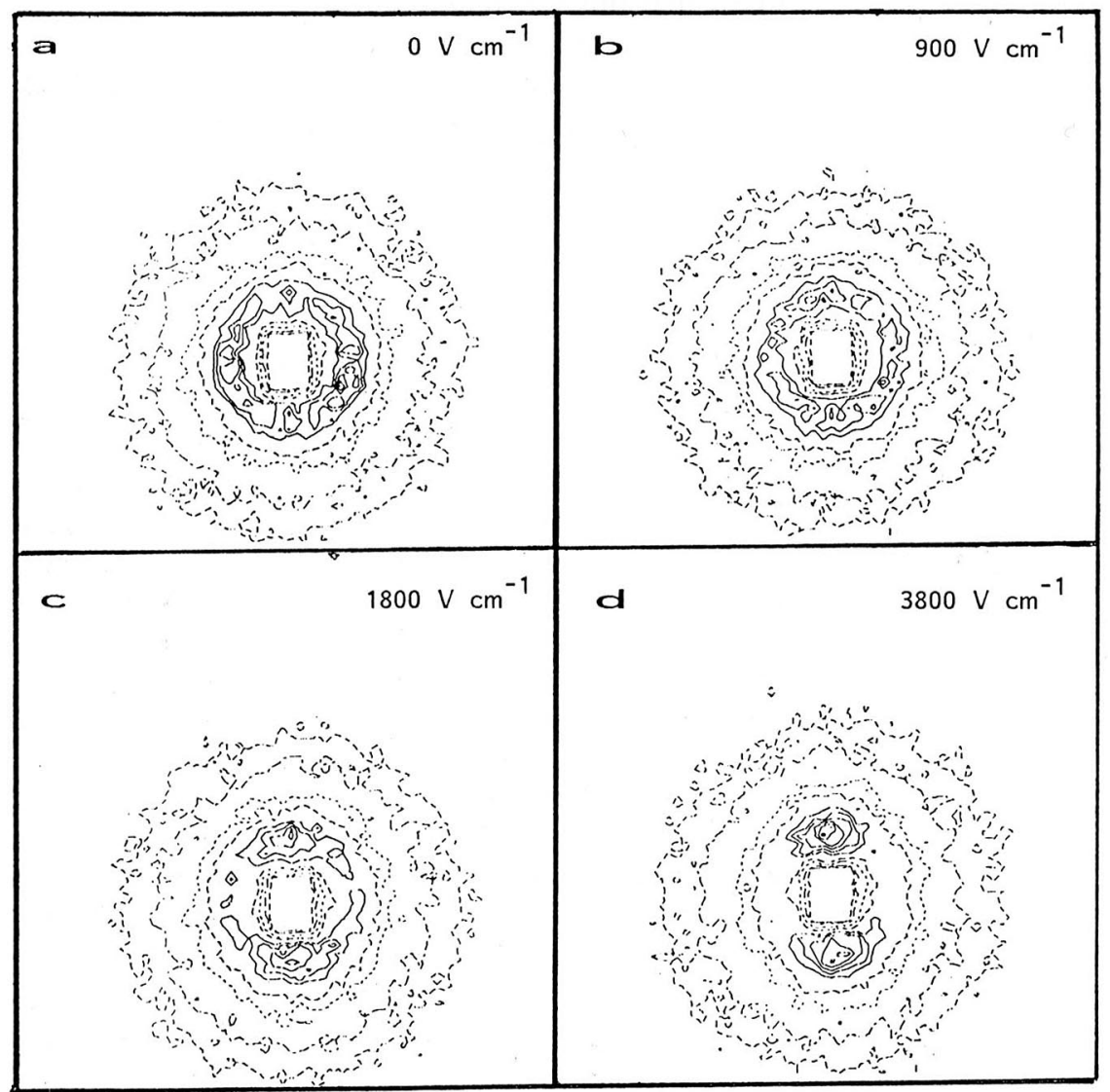

Fig. 4. Contour plots of constant intensity, $\mathrm{I}(\mathrm{Q})$, lines against $\mathrm{Q}$ for a $10 \% \mathrm{w} / \mathrm{w}$ poly(methylmethacrylate) dispersion in dodecane containing $0.025 \%$ calcium octnoate at various applied field strengths 


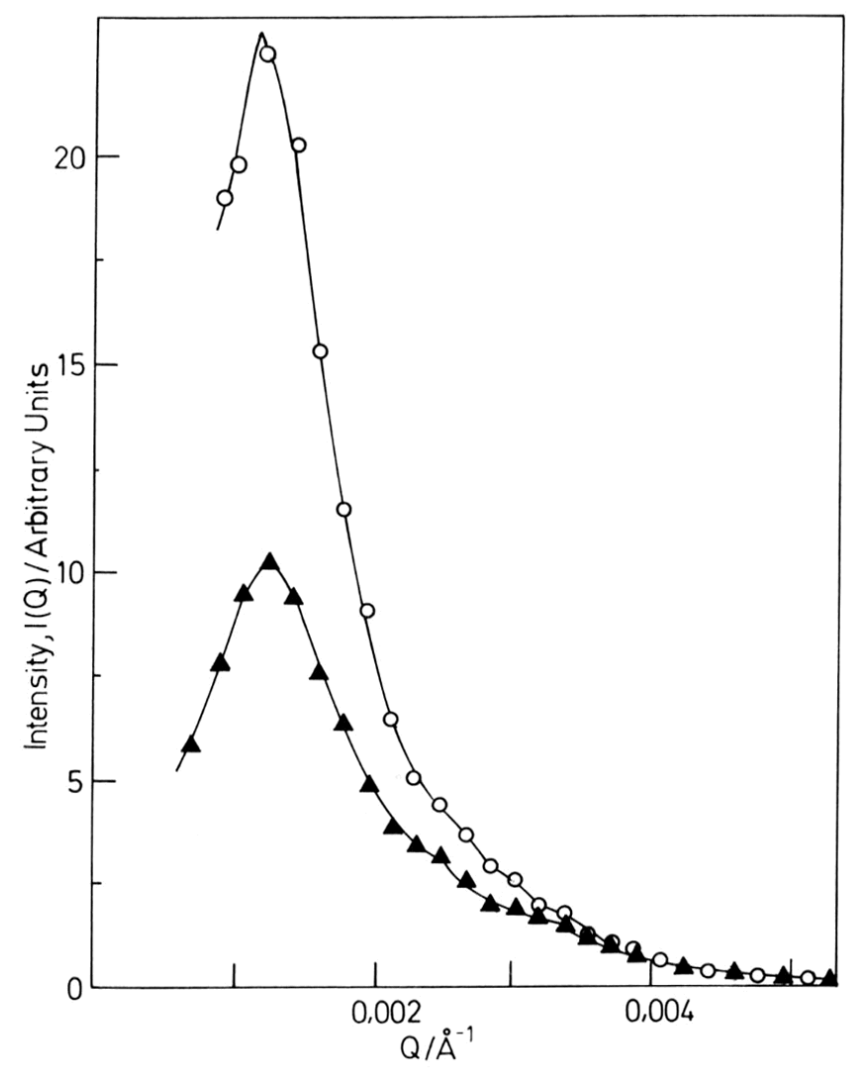

Fig. 5. Intensity, $\mathrm{I}(\mathrm{Q})$, against scattering vector, $\mathrm{Q}$, for a $10 \%$ $\mathrm{w} / \mathrm{w}$ poly(methylmethacrylate) dispersion in dodecane containing $0.025 \%$ calcium octanoate at a field strength of $3800 \mathrm{~V} \mathrm{~cm}^{-1}$. - O-, perpendicular to the applied field; parallel to the applied field

Using the information obtained on the 2-dimensional detector, radial averages were taken in sectors at $0^{\circ} \pm 15^{\circ}$ and $90^{\circ} \pm 15^{\circ}$. This gave $\mathrm{I}(\mathrm{Q})$ against $\mathrm{Q}$ plots for the sample in directions parallel and perpendicular to the applied electric field. These are illustrated in Fig. 5. In the direction of the field the intensity has been reduced in comparison to that in the absence of the field. The peak in intensity is very well defined suggesting a significant peak in the curve of $\mathrm{S}(\mathrm{Q})$ against $\mathrm{Q}$ thus indicating strong interaction between the particles. In a direction perpendicular to the applied electric field the intensity has markedly increased compared to that in the absence of the field. The peak is still present, although less well defined, and has moved marginally to a lower $Q$ value.

\section{Discussion}

Three separate pieces of evidence suggest that particles of poly(methylmethacrylate) stabilised by poly(12-hydroxystearic acid) become electrically charged in the presence of low concentrations of cal- cium octanoate in a low relative permittivity medium, dodecane. These are:

i) the formation of a well-defined peak in the curves

of $I(Q)$ against $Q$ indicating a well-defined peak in $S(Q)$ against $Q$.

ii) the development of a positive electrophoretic mobility in the presence of calcium octanoate.

iii) the development of anisotropy, as detected by small angle neutron scattering in the presence of an applied electric field.

The calcium octanoate used in these experiments has a similarity to the calcium diisopropyl salicylate used in earlier work by van der Minnie and Hermanie [12-14]. Since the conductivity of the dodecane increases on the addition of calcium octanoate [15] weak dissociation appears to occur to give,

$$
\mathrm{Ca}(\mathrm{Oct})_{2} \gtrless \mathrm{CaOct}^{+}+\mathrm{Oct}^{-}
$$

and possibly

$$
\mathrm{CaOct}^{+} \gtrless \mathrm{Ca}^{2+}+\mathrm{Oct}^{-} \text {. }
$$

Since the Oct ion is essentially oleophilic, it appears likely that this will remain in the dodecane whilst the $\mathrm{Ca}^{2+}$ or $\mathrm{CaOct}^{+}$associates with the dipole on the ester groups of the poly(12-hydroxystearic acid), leading to a positive charge on the particles by ion-dipole association, viz:

$$
\underset{+\mathrm{C}=\mathrm{O}---\mathrm{Ca}^{2+}}{\longrightarrow}
$$

Only a small number of charges would be needed to produce a positive charge on the particles.

The results shown in Fig. 2 for a $40 \%$ w/w dispersion suggest that in the absence of added calcium octanoate the interaction between the particles is relatively short range consistent with the hard sphere behaviour observed in earlier work [3]. Addition of calcium octanoate in small quantities causes the peak in $I(Q)$ against $Q$ to sharpen considerably suggesting much stronger interaction and short range ordering of the particles, due to electrostatic interactions. The fact that the latter are of long range is substantiated by experiments on the $10 \% \mathrm{w} / \mathrm{w}$ dispersion where a well-defined peak is found with calcium octanoate present (Fig. 4a), but is essentially absent for the dispersion without calcium octanoate. On the application of the electric field an interesting effect occurs in that the intensity increases in the direction perpendicular to the field and decreases in the direction parallel 
to the field. This shows clearly with a field of $3800 \mathrm{~V}$ $\mathrm{cm}^{-1}$ as illustrated in Fig. 5. In the direction of the field the peak is very sharp. It is somewhat less so in the perpendicular direction and has moved slightly to a lower $Q$ value. The complete interpretation of these results requires further analysis and separation of the curves into interactions $(\mathrm{S}(\mathrm{Q}))$ and form factors $(\mathrm{P}(\mathrm{Q}))$. A possible interpretation of the results is that the dipoles formed by the charges on the particles line up in the electric field and cause a preferential alignment in the direction of the field which causes increased interaction in that direction without essentially changing the number concentration, hence sharpening $S(Q)$. In view of the sensitivity of the $\mathrm{I}(\mathrm{Q})$ against $\mathrm{Q}$ curves to the rapid falling off of $P(Q)$ with $Q$ this could lead to a major drop in intensity. Similarly, a weaker interaction in the perpendicular direction at the same number concentration would diminish the structure and hence give an increase in intensity.

A measurement of electrophoretic mobility on a very dilute dispersion in the presence of calcium octanoate gave a value of $0.1 \mu \mathrm{m} \mathrm{cm} \mathrm{V} \mathrm{V}^{-1}$ and hence the time for the particle to travel one particle radius $(0.1 \mu \mathrm{m})$ in a field of $3800 \mathrm{~V} \mathrm{~cm}^{-1}$ can be calculated to be $0.3 \mathrm{msec}$. A similar calculation for the particle to diffuse translationally one radius gives the time as $0.7 \mathrm{msec}$. Hence the ratio;

\section{time to travel one radius in the electric field time to diffuse one radius}

gives a value of 0.43 . This rather simplistic calculation would suggest that appreciable Brownian motion of the particles is still occurring even in the presence of the electric field of $3800 \mathrm{~V} \mathrm{~cm}^{-1}$. This would suggest that the average number concentrations in the directions parallel and perpendicular to the field are probably not appreciably different, although the particleparticle interactions, as suggested above, are significantly different.

\section{Acknowledgements}

We wish to acknowledge with thanks the continued support of the Science and Engineering Research Council and the Institute Laue Langevin for neutron facilities.

\section{References}

1. Barrett KEJ (1975) Dispersion Polymerisation in OrGanic Media, John Wiley and Sons, Inc., London

2. Antl L, Goodwin JW, Hill RD, Ottewill RH, Owen SM, Papworth S, Waters J (1986) Colloids Surfaces 17:67

3. Markovic I, Ottewill RH, Underwood SM, Tadros TF (1986) Langmuir 2:625

4. Ottewill RH, (1989) Langmuir 5:4

5. Lindner P, Markovic I, Oberthür RC, Ottewill RH, Rennie AR (1988) Prog Colloid Polym Sci 76:47

6. Ashdown S, Markovic I, Ottewill RH, Lindner P, Oberthür RC, Rennie AR (1990) Langmuir 6:303

7. Rennie AR, Ashdown S, Lindner P, Markovic I, Oberthür RC, Ottewill RH, (1990) Polymer Colloids NATO Advanced Study Institute, Strasbourg, Kluwer

8. Ottewill RH, Rennie AR, (1989) J Applied Hydrodynamics, in press

9. Ackerson BJ, Hayter JB, Clark NA, Cotter L (1986) J Chem Phys 84:2344

10. (1988) Neutron Beam Facilities at the High Flux Reactor, Institut Laue Langevin, Grenoble

11. Ghosh RE (1989) A Computing Guide for Small Angle Scattering Experiments, Institut Laue Langevin, 89 GH $02 \mathrm{~T}$

12. van der Minnie JL, Hermaine PHJ (1952) J Colloid Sci 7:600

13. van der Minnie JL, Hermaine PHJ (1953) J Colloid Sci $8: 38$

14. Klinkenberg AK, van der Minnie JL (1958) Electrostatics in the Petroleum Industry, Elsevier, Amsterdam

15. Markovic I, Ottewill RH, (1989) to be published

Authors' address

Prof. Dr. R. H. Ottewill

School of Chemsitry

University of Bristol

Cantock's Close

Bristol, BS8 1TS, England 